\title{
General Psychiatry Consensus on potential biomarkers developed for use in clinical tests for schizophrenia
}

Ping Lin, ${ }^{1,2}$ Junyu Sun, ${ }^{3}$ Xiaoyan Lou, ${ }^{1}$ Dan Li, ${ }^{1}$ Yun Shi, ${ }^{1}$ Zhenhua Li, ${ }^{1}$ Peijun Ma, ${ }^{1}$ Ping Li, ${ }^{1}$ Shuzi Chen, ${ }^{1}$ Weifeng Jin, ${ }^{1}$ Shuai Liu, ${ }^{1}$ Qing Chen, ${ }^{1}$ Qiong Gao, ${ }^{1}$ Lili Zhu, Jie Xu, ${ }^{1}$ Mengyuan Zhu, ${ }^{1}$ Mengxia Wang, ${ }^{1}$ Kangyi Liang, ${ }^{1}$ Ling Zhao, ${ }^{1}$ Huabin Xu, ${ }^{4}$ Ke Dong, ${ }^{5}$ Qingtian Li, ${ }^{5}$ Xunjia Cheng, ${ }^{2}$ Jinghong Chen (i) , 6,7 Xiaokui Guo ${ }^{5}$
To cite: Lin P, Sun J, Lou X, et al. Consensus on potential biomarkers developed for use in clinical tests for schizophrenia. General Psychiatry 2022;35:e100685. doi:10.1136/ gpsych-2021-100685

- Additional supplemental material is published online only. To view, please visit the journal online (http://dx.doi.org/10.1136/ gpsych-2021-100685).

$\mathrm{PL}, \mathrm{JS}$ and $\mathrm{XL}$ are joint first authors.

Received 22 0ctober 2021 Accepted 07 January 2022

Check for updates

(C) Author(s) (or their employer(s)) 2022. Re-use permitted under CC BY-NC. No commercial re-use. See rights and permissions. Published by BMJ.

For numbered affiliations see end of article.

Correspondence to

Dr Jinghong Chen

chenjh_008@hotmail.com

Professor Xiaokui Guo; xkguo@shsmu.edu.cn

Professor Xunjia Cheng; xjcheng@shmu.edu.cn

\section{ABSTRACT}

Background Schizophrenia is a serious mental illness affecting approximately 20 million individuals globally. Both genetic and environmental factors contribute to the illness. If left undiagnosed and untreated, schizophrenia results in impaired social function, repeated hospital admissions, reduced quality of life and decreased life expectancy. Clinical diagnosis largely relies on subjective evidence, including self-reported experiences, and reported behavioural abnormalities followed by psychiatric evaluation. In addition, psychoses may occur along with other conditions, and the symptoms are often episodic and transient, posing a significant challenge to the precision of diagnosis. Therefore, objective, specific tests using biomarkers are urgently needed for differential diagnosis of schizophrenia in clinical practice.

Aims We aimed to provide evidence-based and consensus-based recommendations, with a summary of laboratory measurements that could potentially be used as biomarkers for schizophrenia, and to discuss directions for future research.

Methods We searched publications within the last 10 years with the following keywords: 'schizophrenia', 'gene', 'inflammation', 'neurotransmitter', 'protein marker', 'gut microbiota', 'pharmacogenomics' and 'biomarker'. A draft of the consensus was discussed and agreed on by all authors at a round table session.

Results We summarised the characteristics of candidate diagnostic markers for schizophrenia, including genetic, inflammatory, neurotransmitter, peripheral protein, pharmacogenomic and gut microbiota markers. We also proposed a novel laboratory process for diagnosing schizophrenia in clinical practice based on the evidence summarised in this paper.

Conclusions Further efforts are needed to identify schizophrenia-specific genetic and epigenetic markers for precise diagnosis, differential diagnosis and ethnicity-specific markers for the Chinese population. The development of novel laboratory techniques is making it possible to use these biomarkers clinically to diagnose disease.

\section{INTRODUCTION}

Schizophrenia is a chronic disorder characterised by continuous or relapsing episodes of psychosis, with hallucinations, delusions
Key messages

What is already known on this topic

- Schizophrenia is a complex mental illness. The precise and early diagnosis of schizophrenia is challenging.

What this study adds

- This study includes a comprehensive introduction of the characteristics of candidate diagnostic markers for schizophrenia and a novel laboratory process for diagnosing schizophrenia in clinical practice.

How this study might affect research, practice or policy

- This study provides a first-hand laboratory testing basis for the clinical diagnosis of schizophrenia.

and disorganised thinking as major manifestations. ${ }^{1}$ Schizophrenia has a lifetime prevalence of approximately $0.3 \%-0.7 \%^{2}$ and mainly affects teenagers and young adults and more males than females (the male-to-female ratio is approximately $1.4: 1) .^{3}$ Other risk factors include advanced paternal age, perinatal events, influenza or other infections in the second trimester, birth in spring and drug use. ${ }^{4-6}$ The clinical diagnosis of schizophrenia is based on the criteria in the Diagnostic and Statistical Manual of Mental Disorders edition 5 or the International Statistical Classification of Diseases and Related Health Problems edition 11. For schizophrenia, the presence or absence of specific first-rank positive symptoms (including auditory hallucinations, thought withdrawal, insertion or interruption; thought broadcasting; somatic hallucination; delusional perception; feelings or actions of being controlled by external agents) may be particularly helpful for clinical diagnosis. ${ }^{7}$ However, negative symptoms (including affective flattening, avolition, alogia and anhedonia) should not be neglected. Although 
they are less prominent during the acute phase, they reflect diminished emotional expression and reduced motivation and behavioural activities. ${ }^{8}$

The precise and early diagnosis of schizophrenia is challenging because the pathological mechanisms of the disease are not fully understood. The current diagnostic criteria are based on subjective evidence; however, psychotic episodes are transient and difficult to detect, especially during the early stage of the disease. Therefore, objective and specific biomarkers by laboratory testing are urgently needed in the clinical diagnosis of schizophrenia. In general, these biomarkers are classified into three categories: (i) persistent markers such as genetic markers, (ii) episodic and symptom-related markers such as inflammatory markers and (iii) markers as indicators of treatment responsiveness, such as pharmacogenomic markers. The major goals of testing these biomarkers are to (i) identify the risk of schizophrenia to benefit early diagnosis and intervention, (ii) assist in diagnosis and differential diagnosis, (iii) evaluate disease severity and progression, (iv) predict suicide risk and (v) assist in individualised treatment strategies by testing markers related to drug efficacy and side effects.

The objectives of this study were to (i) discuss the existing evidence for laboratory measurements as potential diagnostic tools, (ii) propose a novel laboratory process for the recognition of schizophrenia for use in clinical practice and (iii) suggest the design of laboratory and clinical studies by outlining the further investigations required. We hoped to comprehensively summarise the currently available approaches and trigger more clinical research to improve diagnostic tools and technologies.

\section{METHODS}

We searched PubMed, Embase and the Cochrane Library for publications within the last 10 years with the following keywords: 'schizophrenia', 'gene', 'inflammation', 'neurotransmitter', 'protein marker', 'gut microbiota', 'pharmacogenomics' and 'biomarker'. An initial draft for circulation was developed by the Shanghai Mental Health Center and subsequently discussed at a round table session at the 2021 Annual Meeting of Laboratory Medicine of the Chinese Medical Doctor Association held between 12 and 14 May 2021.

\section{Existing evidence for laboratory measurements as potential diagnostic tools \\ Genetic markers}

Schizophrenia has strong heritability, estimated to be between $70 \%$ and $80 \% .{ }^{9}$ Having a first-degree relative with the disease has been considered the greatest risk factor for developing schizophrenia, and over $40 \%$ of identical twins of those with schizophrenia are also affected. ${ }^{10}$ Previous studies have shown that a higher genetic risk of schizophrenia is associated with worse cognitive performance, ${ }^{11}$ and early-onset cases appear to be associated with a higher rate of large cytogenetic abnormalities and rare structural variants than those reported in late-onset cases. $^{12}$

Copy number variants (CNVs) have been considered to induce greater risk, and individuals with schizophrenia have an increased genome-wide CNV burden, ${ }^{13}$ although such changes have been found in only $2 \%-3 \%$ of patients with schizophrenia. ${ }^{14}$ One of the most recognised CNVs is a deletion at $22 q 11.2$, which has been estimated to have a prevalence of $1 \%-2 \%$ in schizophrenia cases and is also seen in early-onset cases (22q11.2 deletion syndrome is also known as the velocardiofacial syndrome).$^{15-17}$ In addition, it has been estimated that up to $25 \%$ of adults with the deletion have schizophrenia, ${ }^{18}$ and the deletion induces a $30 \%$ lifetime risk for schizophrenia. ${ }^{19}$ In conclusion, the deletion at 22q11.2 may play a crucial role in disease development and progression. Other CNVs, such as deletions at $17 \mathrm{q} 12$, duplications at $16 \mathrm{p} 11.2$ and deletions at 15q11.2, have also been reported to increase the risk of developing schizophrenia and are often comorbid with autism and intellectual disabilities. ${ }^{17}{ }^{20}$ In terms of ethnic factors, genetic studies comparing large East Asian cohorts with European cohorts revealed similarities in the effects of common genetic variants on the risk of schizophrenia. ${ }^{21}$

Although individual loci are considered to have relatively minor effects, an association between these loci and schizophrenia symptoms has been established, and schizophrenia is considered highly polygenic. Large cohort genome-wide association studies (GWAS) have identified $>100$ loci that are associated with schizophrenia, including loci in major histocompatibility complex (MHC) regions, microRNA-137 (MIR137), zinc-finger protein 804A (ZNF804A), neurogranin (NRGN) and transcription factor $4(T C F 4)^{22-24}$; among these associations are enriched within genes expressed in tissues that play critical roles in immunity, particularly B-lymphocyte lineages involved in acquired immunity, providing evidence for the hypothesised link between the immune system and schizophrenia. ${ }^{22}$ The identified loci overlap and vary in different ethnicities. For instance, studies on cohorts with European ancestry or a combination of European and African ancestry revealed schizophrenia-related singlenucleotide polymorphisms (SNPs) for TRIM26, CCDC68, TCG4, MIR137, DPYD-MIR137, PCGEM1, CSMD1, MMP16, CNNM2, NT5C2, STT3A, AMBRA1, DGKZ, CHRM4, MDK, ITIH3, ITIH3-ITIH4, AS3MT, calcium channel subunits $C A C N A 1 C$ and $C A C N B 2$, human leucocyte antigen (HLA)$C^{*} 01: 02, H L A-D R B 9$ and other multiple loci in the MHC region; SDCCAG8, C10orf26, NRGN, ZNF804A, ankyrin-3 (ANK3), hepatocyte nuclear factor 4y (HNF4G), NADH dehydrogenase (ubiquinone) iron-sulfur protein 4 (NDUFS4), histone deacetylase 9 (HDAC9) and various other loci. ${ }^{11}{ }^{13}{ }^{24-32}$ In addition to some of the previously identified loci, one study in patients with Chinese ancestry revealed four novel loci at 3p21.31, 6q21, 6q27 and $7 \mathrm{q} 31.1 .{ }^{33}$ However, a study in a Japanese cohort did not show any locus of genome-wide significance but supported a polygenic risk for schizophrenia. ${ }^{34}$ 
Some of these loci have been associated with genes that contribute to disease manifestation. For instance, rs830786 within $H N F 4 G$ has been associated with attention/vigilance, and rs67017972 near NDUFS4 has been associated with verbal memory, whereas rs76872642 within HDAC9 has been associated with reasoning and problem-solving. ${ }^{11}$ In addition, $A N K 3$ variants have been associated with lower cognitive performance, with the risk allele rs1938526 particularly associated with the domains of verbal memory, working memory and attention, ${ }^{35}$ whereas $N R G N$ variants have been associated with episodic and working memory. ${ }^{36}$ One study suggested the involvement of rs6994992 within neuregulin 1 in the susceptibility to developing cognitive deficits in patients at the first stage. ${ }^{37}$ Variants of GRM5 (coding for metabotropic glutamate receptor 5 (mGluR5)) have been shown to be associated with cognitive impairment. ${ }^{38}$ Additionally, SNPs in dystrobrevin-binding protein 1 have been associated with a variety of cognitive domains in schizophrenia, including attention, vigilance, memory and processing speed. ${ }^{39}{ }^{40}$ Furthermore, genes related to neurotransmitters have also been linked to cognitive impairment in schizophrenia. For instance, the 5-hydroxytryptamine 2A (5-HT2A) receptor gene polymorphism is associated with sustained attentional impairment in patients with earlyonset schizophrenia. ${ }^{41}$ Moreover, the dopamine $\beta$-hydroxylase $(\mathrm{D} \beta \mathrm{H})$ polymorphism may be linked to $\mathrm{D} \beta \mathrm{H}$ activity in the Chinese population and may influence some aspects of cognitive function in schizophrenia. ${ }^{42}$

Some of these loci have been associated with changes in brain structures. Genetic variations of CACNA1C, NRGN, TCF4 and ZNF804A have been associated with grey matter volume, and variations in ANK3 and ZNF804A have been associated with white matter integrity. ${ }^{36}$ Variations in $A N K 3$ have been linked to the brain structure, and NRGN variations have been linked to brain volume. ${ }^{36}$ Specifically, the risk allele of ANK3 (rs1938526) is associated with widespread cortical thinning in patients with firstepisode psychosis, ${ }^{35}$ and GRM5 variants are associated with a reduction in right hippocampal volume in schizophrenia without affecting mGluR5 protein expression. ${ }^{38}$

Other loci have also been associated with potential mechanisms. For instance, ANK3, CACNA1C, diacylglycerol kinase eta, NRGN and ZNF804A have all been associated with regional activation during executive tasks, and CACNA1C and ZNF804A are associated with functional connectivity during executive tasks. ${ }^{36}$ MIR137 plays an essential role in synaptic plasticity, ${ }^{43}$ and some of its targets include GWAS-identified schizophrenia genes. ${ }^{445}$ The MHC region, which encodes genes critical to immunity, is involved in susceptibility to schizophrenia, and risk associated with NRGN and TCF4 points to perturbation of pathways involved in brain development, memory and cognition. ${ }^{30}{ }^{46}$ The catechol-O-methyltransferase gene Val allele slightly increases the risk of schizophrenia with a potential mechanism involving increasing prefrontal dopamine catabolism, thus impairing prefrontal cognition and physiology. ${ }^{47}$ Furthermore, these genomic loci may also be present in specific neuronal cell types. For instance, single-cell RNA sequencing has revealed that common variants are consistently present in pyramidal neurons, medium spiny neurons and specific interneurons, but to a much lesser extent in glial cells, indicating specific roles they may play in the disease mechanisms. ${ }^{48}$

Interestingly, schizophrenia has been shown to share some common risk variants with other psychiatric diseases, with the highest SNP co-heritability between schizophrenia and bipolar disorder, followed by schizophrenia and major depressive disorder, suggesting potential common pathways among different psychiatric diseases. $^{49}$

Environmental factors, which have been linked to changes in gene expression via epigenetic modulation, have also been considered risk factors for developing schizophrenia. Previous analyses of gene methylation in schizophrenia have mainly focused on neurotransmitters, such as $\gamma$-aminobutyric acid (GABA), glutamate, serotonin and dopamine, and various candidate genes have been identified by analysis of brain tissues, whole blood and saliva. ${ }^{50}$ DNA methylation of the reelin and glutamic acid decarboxylase genes (RELNand GAD1, respectively), which are involved in the GABAergic pathway, has been identified in the brains of patients with schizophrenia, ${ }^{5152}$ whereas DNA methylation of the serotonin receptor type-1 gene (HTR1A) is increased in the peripheral blood of patients with schizophrenia compared with controls. ${ }^{53}$ Notably, one study has revealed that the most significant differentially methylated positions in the Chinese cohort are the C17orf63, THAP domain-containing protein 1 and potassium voltage-gated channel subfamily KQT member 4 genes. ${ }^{54}$

In addition to DNA methylation, changes in microRNA (miRNA) expression are observed in postmortem brains of patients with schizophrenia. ${ }^{55}$ A previous study identified an increase in miR-328, miR-17-5p, miR-134, miR652 , miR-382 and miR-107 in the brains of patients with schizophrenia. ${ }^{56}$ A meta-analysis of studies on peripheral blood mononuclear cells revealed decent sensitivities and specificities of miR-181b-5p, miR-21-5p, miR-195-5p, miR-137, miR-346 and miR-341-5p for the diagnosis of schizophrenia. ${ }^{57}$

\section{Inflammatory markers}

Inflammation is considered to play a crucial role in the pathogenesis of schizophrenia. A postmortem study revealed decreased interleukin (IL)- $1 \alpha$ and interferon (IFN)--y-inducible protein 10 expression as well as increased IFN- $\alpha$ expression in the superior temporal gyrus of patients with schizophrenia ${ }^{58}$ suggesting a direct association of inflammatory markers with the disease. Since then, a variety of inflammatory markers have been assessed in different types of biospecimens, including peripheral blood and cerebrospinal fluid (CSF).

$\mathrm{C}$ reactive protein (CRP) has been shown to increase in schizophrenia and to be associated with disease severity. ${ }^{59}$ A meta-analysis revealed that CRP is 
significantly associated with cognitive function in schizophrenia. ${ }^{61}$ In addition, serum levels of IL-1RA, IL-6, IL-7, IL-8, IL-9, IL-10, IL-13, IFN-y, eotaxin-1, granulocytemacrophage colony-stimulating factor, monocyte chemoattractant protein 1 (MCP-1, also known as CCL2), platelet-derived growth factor subunit $\mathrm{B}$, monocyte inflammatory proteins (MIP-1 $\alpha$ and MIP-1 $\beta$ ), vascular endothelial growth factor $\mathrm{A}$ and regulated on activation, normal $\mathrm{T}$ cell expressed and secreted (also known as CCL5) have all been shown to increase in patients with multi-episode schizophrenia. ${ }^{62}$ In addition, a cytokine imbalance of $\mathrm{T}$ helper types 1 and 2 has also been reported to be present in peripheral blood as well as in CSF. ${ }^{63}$ Furthermore, CSF levels of IL- 6 and IL- 8 have been shown to be elevated in schizophrenia. ${ }^{64}$ These inflammatory markers may also be able to predict the prognosis of schizophrenia, as higher baseline blood IL-6 is correlated with a greater reduction in the Positive and Negative Syndrome Scale (PANSS) total and general subscale scores at 3 and 6 months and PANSS negative subscale scores at 3 months. ${ }^{65}$ Therefore, changes in these inflammatory markers may potentially serve to predict disease progression and manifestation.

In addition, changes in these inflammatory markers have been shown to be directly associated with the disease mechanisms. For instance, it has been hypothesised that inflammation interferes with cellular pathways, inducing the metabolism of tryptophan to kynurenic acid in schizophrenia. This is supported by evidence that the plasma level of kynurenine is positively correlated with IL-1 $\beta$ and PANSS, ${ }^{66}$ and the elevation of IL- 6 and tumour necrosis factor- $\alpha$ is associated with the kynurenine pathway. ${ }^{67}$

Importantly, these inflammatory markers may predict disease progression and drug efficiency. A meta-analysis has shown that the elevation of IL-1 $\beta$, IL- 6 and transforming growth factor- $\beta$ in acutely relapsed patients and first-episode psychosis could be normalised by antipsychotic treatment, whereas the elevation of IL-12, (IFN$\gamma)$, TNF- $\alpha$ and soluble IL-2 receptor (sIL-2R) in acute exacerbations is not reversible following antipsychotic treatment. ${ }^{68}$ Therefore, inflammatory markers serve as important biomarkers for the prognosis of disease progression and treatment response in schizophrenia.

Apart from changes in these inflammatory markers, autoimmune dysfunction and maternal/perinatal infections have also been considered risk factors for schizophrenia. ${ }^{69}$ Patients with schizophrenia who have a first-degree relative with schizophrenia are more likely to have a parent or sibling with an autoimmune disease, ${ }^{70}$ and a history of autoimmune diseases increases the risk of developing schizophrenia by $29 \% .^{71}$ Increased risks of systemic lupus erythematosus, Graves' disease, coeliac disease and various other autoimmune diseases have been reported in patients with schizophrenia ${ }^{72}{ }^{73}$; however, a reverse association with rheumatoid arthritis is also observed. ${ }^{72} 74$

\section{Neurotransmitter markers}

Three main theories have been put forth for the pathophysiology of psychosis: serotonin theory, dopamine theory and glutamate theory (N-methyl-D-aspartate hypoactivity). ${ }^{75}$ The serotonin theory proposes that increased serotonin or 5-HT2A receptor activity leads to psychosis. The dopamine theory proposes that dopamine is hyperactive in the dopaminergic pathway at dopamine D2 receptors, causing positive symptoms, which is further supported by the fact that all antipsychotics target blockage of $\mathrm{D} 2$ receptors. ${ }^{76}$ The glutamate theory proposes that the N-methyl-D-aspartate receptor is less functional in the prefrontal cortex. ${ }^{75}$

An early study revealed that administration of lysergic acid diethylamide, a 5-HT antagonist, induced hallucinations, raising the hypothesis that 5-HT deficiency may contribute to the pathogenesis of schizophrenia. ${ }^{75}$ A previous positron emission tomography study on firstepisode, drug-naïve patients with schizophrenia revealed lower 5-HT2A receptor binding in the frontal cortex, and 5-HT2A binding was negatively correlated with positive psychotic symptoms in male patients. ${ }^{77} 78$ Therefore, the mechanism may be related to reduced binding of 5-HT to the receptor rather than an absolute decrease in 5-HT. This is further supported by subsequent postmortem studies that revealed elevated 5-HT and/or its primary metabolite 5-hydroxyindoleacetic acid (5-HIAA) in specific brain regions, although controversial results have been reported. ${ }^{79}$ In the meantime, CSF levels of 5-HIAA have been revealed to be negatively associated with delusions and sadness, as assessed by the Comprehensive Psychopathological Rating Scale but not with suicide attempts. ${ }^{80} 81$

Early clinical studies also revealed that an amphetamine challenge induced increased dopamine release accompanied by worsening of psychotic symptoms ${ }^{82}$ and administration of antipsychotic drugs that block D2 dopamine receptors reduced the severity of prodromal symptoms, ${ }^{83}$ which indicated that dopamine dysregulation might contribute to the development of psychosis. This concept is further supported by a postmortem study showing the elevation of dopamine and its receptors in brain subregions, including striata, nucleus accumbens and caudate of patients with schizophrenia. ${ }^{84}$ However, a more recent imaging study revealed that reduced dopamine was present in striata and the dorsolateral prefrontal cortex on amphetamine challenge. ${ }^{85}$ Furthermore, CSF levels of homovanillic acid, a dopamine metabolite, are decreased in patients with schizophrenia compared with controls. ${ }^{86}$ Other indirect evidence showed that tyrosine hydroxylase, the rate-limiting enzyme for dopamine synthesis, is elevated in the substantia nigra in patients with schizophrenia compared with healthy controls. ${ }^{87}$ In addition, in vivo imaging analysis revealed significant elevation of dopaminergic function in presynapses of the schizophrenia brain, and that the change in presynaptic dopamine function was more consistent in acute psychosis than in chronic disease processes. ${ }^{88} 89$ Other imaging studies 
have revealed that the uptake of ${ }^{18} \mathrm{~F}$-dopa is elevated in the striatum and correlated with the severity of psychopathological and neuropsychological impairment in the prodromal phase of the disease. ${ }^{90}$

Glutamate is considered to play a crucial role in the pathophysiology of schizophrenia. Previous meta-analyses on proton magnetic resonance spectroscopy (MRS) studies revealed significant increases in glutamate and glutamine (Glx) in several brain regions, including the basal ganglia, thalamus and medial temporal lobe. ${ }^{91}$ In contrast, individual MRS studies revealed reduced levels of glutathione-glutamate components in the anterior cingulate cortex in patients with stable schizophrenia. ${ }^{92}$ In the periphery, lower plasma levels of glutathione have been shown in patients with schizophrenia and are positively correlated with Glx levels in the anterior singulate cortex as assessed by MRS. ${ }^{93}$

In addition, other neurotransmitters, such as norepinephrine (NE), have been shown to decrease in serum in schizophrenia compared with controls, and overnight urine levels of $\mathrm{NE}$ are negatively correlated with working memory in schizophrenia. ${ }^{94} 95$

At present, most of the studies are established by in vivo imaging or on postmortem brain tissue and occasionally in the CSF. Assessment of neurotransmitters in peripheral biofluids, particularly in the blood, is lacking.

\section{Peripheral protein and metabolite markers}

Various peripheral proteins have been assessed in schizophrenia, including nerve growth factor (NGF), brainderived neurotrophic factor (BDNF), homocysteine (Hcy), vitamin B and G72.

NGF is a neurotrophic protein that regulates various physiological functions. Meta-analyses have revealed that serum and plasma levels of NGF are decreased in both drug-naïve and medicated patients with schizophrenia, independent of demographic factors such as age and sex. ${ }^{96-98}$ This is confirmed by another study of drug-naive first-episode patients with schizophrenia, indicating that NGF is a stable marker for schizophrenia. ${ }^{99}$ Although serum NGF levels did not differ between schizophrenia and controls in a different study, it was still correlated with grey matter volume in regions such as the left prefrontal lobe, left midcingulate cortex and brainstem in schizophrenia. ${ }^{100}$

BDNF is an important neurotrophic factor in the brain. A meta-analysis of drug-naive first-episode patients with schizophrenia revealed decreased levels of BDNF in the peripheral blood. ${ }^{99}$ An individual study also revealed lower plasma levels of BDNF in schizophrenia and the correlation of BDNF with certain cognitive dimensions, such as memory. ${ }^{101}$ In addition, patients with schizophrenia and depression present lower plasma levels of BDNF than patients with schizophrenia but without depression, who have lower BDNF levels than those in controls, indicating its potential for predicting clinical manifestations. ${ }^{102}$ Importantly, BDNF may change continuously from preclinical to clinical stages, as both plasma and serum levels of BDNF are highest in patients with chronic schizophrenia (followed by first-episode patients with psychosis, patients and individuals with an at-risk mental state) but do not predict relapse of schizophrenia, ${ }^{103}$ indicating that BDNF may potentially serve as a longitudinal marker to predict progression from at-risk mental state to schizophrenia. Caution should be taken when setting the threshold of BDNF, as sex may be a factor influencing BDNF levels. A previous study showed that plasma levels of BDNF are significantly higher in female patients with schizophrenia than in male patients. ${ }^{104}$

Hcy is biosynthesised from methionine, and its abnormal elevation leads to imbalanced neurotransmitters (ie, inhibited synthesis of dopamine, NE and 5-HT), resulting in cognitive impairment and negative symptoms of schizophrenia. ${ }^{105}$ Previous studies of a Han Chinese population have shown that the incidence of hyperhomocysteinaemia is more than doubled in patients with schizophrenia compared with controls. ${ }^{106}$ Another study of a cohort from Poland showed a similar increased incidence of hyperhomocysteinaemia. ${ }^{107}$ Serum levels of homocysteine are elevated as early as the first episode in schizophrenia $^{108}$ and are negatively associated with cognitive performance and positively correlated with the Calgary Depression Scale for Schizophrenia. ${ }^{109}$ Furthermore, a Japanese study has revealed that total homocysteinerelated SNPs are associated with schizophrenia. ${ }^{110}$ Together, these findings indicate that Hcy may serve as a convenient biomarker in peripheral blood to predict changes in mood and cognition.

Vitamins are considered crucial for body functions. It has been revealed that the level of vitamin $\mathrm{B}_{6}\left(\mathrm{VitB}_{6}\right)$ is decreased in the peripheral blood of patients with schizophrenia compared with controls. ${ }^{111}{ }^{112}$ This is further supported by a meta-analysis that has shown that lower serum levels of $\mathrm{VitB}_{6}$ are associated with the risk of schizophrenia. ${ }^{113}$ Therefore, VitB $_{6}$ may serve as an early marker to assist in predicting the risk of schizophrenia. Findings regarding vitamin $\mathrm{B}_{12}\left(\mathrm{VitB}_{12}\right)$ are less consistent, with both elevation and reduction of $\mathrm{VitB}_{12}$ levels observed in the peripheral blood of patients with schizophrenia compared with controls. ${ }^{111112114}$

G72 modulates D-amino acid oxidase, which degrades D-serine, which is considered to contribute to glutamate neurotransmission. ${ }^{115}$ Models built by machine learning revealed that the G72 protein may serve as a potential biomarker for identifying schizophrenia. ${ }^{116}$ This is consistent with the finding that serum levels of G72 are increased significantly in patients with schizophrenia compared with controls. ${ }^{117}$ However, CSF analysis failed to show a significant change in G72 levels in patients with schizophrenia compared with controls. ${ }^{115}$ Furthermore, a meta-analysis revealed that the T-allele of a D-amino acid oxidase activator SNP was associated with schizophrenia in multiple populations, with the G-allele also associated with schizophrenia in an Asian population. ${ }^{118}$ 


\section{Gut microbiota and their metabolites}

Schizophrenia is associated with reduced microbial diversity and shows a different global community compared with non-psychiatric controls. ${ }^{119}$ A previous study showed that patients with schizophrenia present more anaerobes. ${ }^{120}$ At the phylum level, 16S rRNA sequencing revealed that patients with schizophrenia present a higher abundance of Proteobacteria compared with controls. ${ }^{121}$ However, contrary results are reported in another study showing a reduction of Proteobacteria in schizophrenia. ${ }^{122}$ At the family level, the relative abundance of Christensenellaceae, Enterobacteriaceae and Victivallaceae is increased, whereas that of Pasteurellaceae, Turicibacteraceae, Peptostreptococcaceae, Veillonellaceae, Succinivibrionaceae, Alcaligenaceae, Enterococcaceae, Leuconostocaceae, Rhodocyclaceae and Rikenellaceae is decreased in patients with schizophrenia compared with controls. ${ }^{123} 124$ At the genus level, $a$ and $\beta$ diversities are increased in schizophrenia. ${ }^{120}$ The relative abundance of Succinivibrio, Megasphaera, Collinsella, Klebsiella, Methanobrevibacter and Anaerococcus is increased, whereas that of Blautia, Coprococcus, Roseburia, Haemophilus, Sutterella and Clostridium is decreased in schizophrenia. ${ }^{121} 122$ At the species level, an increased relative abundance of Akkermansia muciniphila, Bifidobacterium adolescentis, Clostridium perfringens, Lactobacillus gasseri, Megasphaera elsdeniis and Clostridium coccoides and a decreased relative abundance of Bifidobacterium spp, Escherichia coli and Lactobacillus spp have been revealed in patients with schizophrenia compared with controls. ${ }^{124} 125$

Alteration of microbial diversity may reflect the presence of disease and predict the risk and disease stage of schizophrenia. For instance, an increase in Enterobacteriaceae has been shown to be potentially related to an increased risk of schizophrenia, whereas Gammaproteobacteria is associated with a lower risk. ${ }^{126}$ In addition, Lachnospiraceae has been associated with chronic schizophrenia. ${ }^{127}$ Furthermore, the gut microbiota has been shown to differentiate between acute schizophrenia and remission. ${ }^{128}$

Few gut microbiota have been linked to clinical symptoms. For instance, Ruminococcaceae is negatively correlated with the severity of negative symptoms, whereas Bacteroides is positively correlated with depressive symptoms. ${ }^{122}$ Deficit schizophrenia has been shown to present elevated IgA levels in response to Hafnei alvei, Pseudomonas aeruginosa, Morganella morganii and Klebsiella pneumoniae compared with non-deficit schizophrenia; more importantly, elevated IgA levels in response to Pseudomonas and elevated IgM levels in response to Gram-negative bacteria are highly predictive of deficit schizophrenia. $^{129}$

In addition to the change in gut bacteria, Candida has also been shown to be present in patients with schizophrenia. Candida albicans seropositivity in schizophrenia has been shown to be correlated with positive psychiatric symptoms, and antibodies against C. albicans decreased significantly following probiotic treatment in male patients with schizophrenia. ${ }^{130}$

\section{Pharmacogenomic markers}

Pharmacogenomic markers are considered crucial in the treatment of schizophrenia. A majority of antipsychotics are metabolised by cytochrome P450 (CYP450), and genetic polymorphisms may result in poor metabolising capacity, subsequently affecting treatment efficacies and causing side effects. A recent consensus supported the use of pharmacogenomic testing for CYP450, CYP2D6 and CYP2C19 genes when developing individualised therapeutic strategies and also suggested testing for human leucocyte antigen genes $H L A-A$ and $H L A-B$ on carbamazepine administration. ${ }^{131}$ In addition, when oxcarbazepine is administered, HLA-B should be tested; when phenytoin is administered, CYP2C9 and HLA-B should be assessed. ${ }^{131}$

In addition to these commonly recognised general genes associated with treatment response, other gene polymorphisms are also important in predicting drug response. A GWAS revealed that higher polygenic risk scores could predict greater post-treatment symptoms, whereas lower polygenic risk scores posed a higher possibility of treatment response. ${ }^{132}$ In a Chinese schizophrenia cohort, polymorphism of the dopamine transporter gene, rs2975226, was associated with allele and genotype frequencies in response to clozapine. ${ }^{133}$ In addition, polymorphism of serotonergic receptor types $2 \mathrm{~A}$, T102C, 2C and 6 has been shown to be correlated with the response to clozapine in schizophrenia. ${ }^{134135}$ Furthermore, a previous study revealed that polymorphisms of $H T R 2 C$ are associated with the treatment response of HTR2C antagonists. ${ }^{136}$

\section{Consensus laboratory process for recognising schizophrenia in clinical practice}

Collectively, we proposed a novel laboratory process for diagnosing schizophrenia (figure 1) based on the evidence summarised in this paper (online supplemental table 1). First, although schizophrenia presents strong heritability, attention needs to be paid when using and interpreting genetic markers for diagnostic purposes. CNVs are significant but rare variations; even for the most recognised disease-specific CNV (deletion at 22q11.2), the estimated prevalence is only $1 \%-2 \%$ in schizophrenia. A large number of schizophreniaassociated CNVs have been identified in GWASs; however, validation is required across multiple studies before their use as biomarkers for schizophrenia. Moreover, co-heritability exists between schizophrenia and other mental disorders, including bipolar disorder and major depressive disorder, posing a challenge for differential diagnosis. Epigenetic modulation has attracted increasing research interest in the development of biomarkers. Specific DNA methylation sites and miRNAs have been proposed as potential biomarkers for schizophrenia. Second, inflammatory markers may serve as important biomarkers for predicting (i) disease progression, (ii) treatment response and drug efficiency and (iii) prognosis. Meanwhile, these inflammatory markers are associated with disease mechanisms and thus may be used as biomarkers for mechanism-related research. Third, neurotransmitter markers could potentially be used to assist 


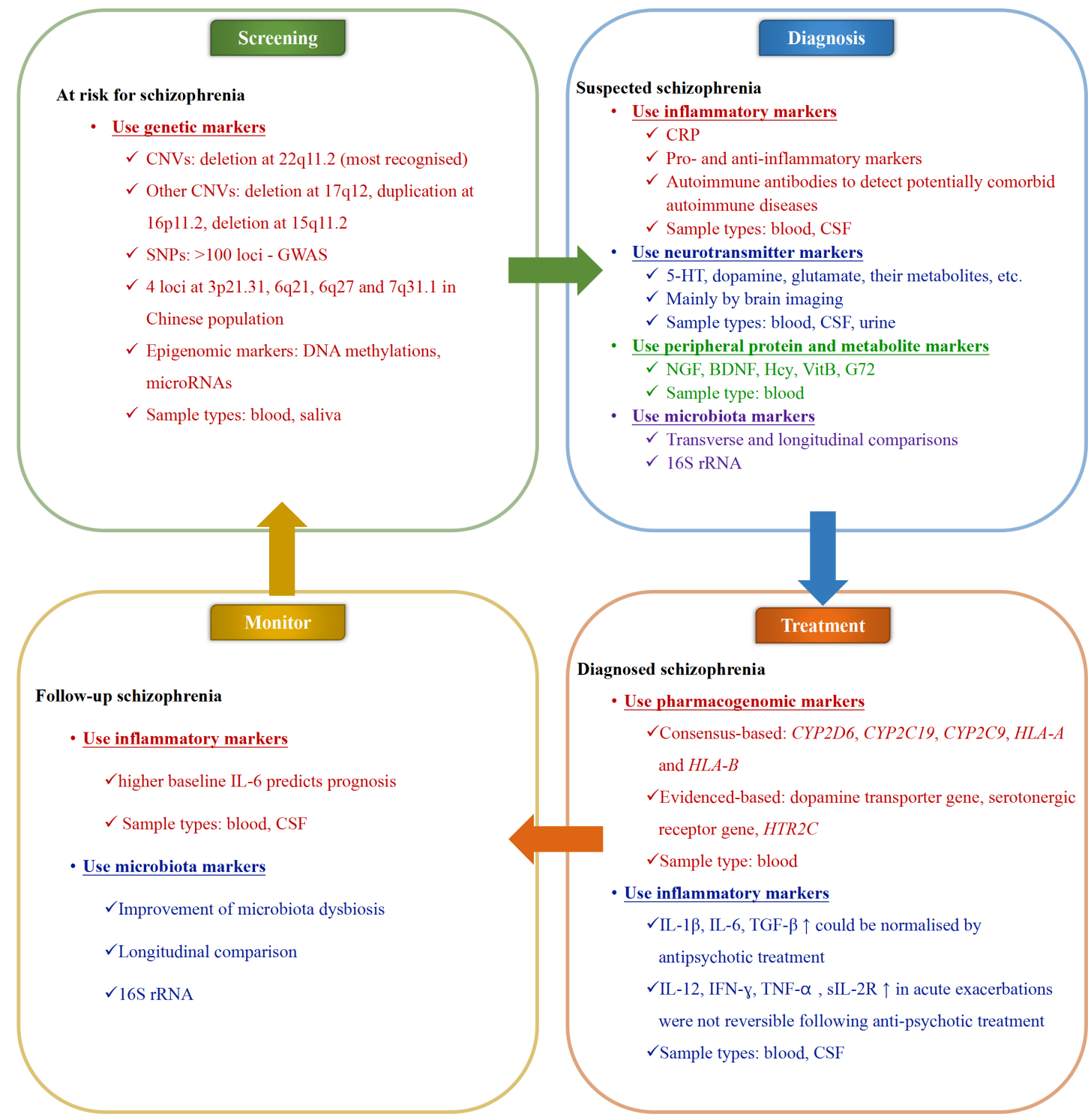

Figure 1 Diagnostic scheme for screening, diagnostic assessments, evaluation of treatment response and postdiagnostic monitoring for schizophrenia. BDNF, brain-derived neurotrophic factor; CNV, copy number variant; CRP, $\mathrm{C}$ reactive protein; CSF, cerebrospinal fluid; GWAS, genome-wide association studies; Hcy, homocysteine; HLA, human leucocyte antigen; 5HT, 5-hydroxytryptamine; IFN, interferon; IL, interleukin; NGF, nerve growth factor; sIL-2R, soluble IL-2 receptor; SNP, singlenucleotide polymorphism; TGF, transforming growth factor; TNF, tumor necrosis factor; VitB, vitamin B.

with the diagnosis of schizophrenia; however, the measurements are mainly performed using imaging analysis, which limits their use in clinical practice. Fourth, peripheral protein markers such as NGF and BDNF could potentially act as stable and longitudinal markers for schizophrenia because changes in these markers are independent of demographic factors and exist continuously from preclinical to clinical stages. In addition, these protein and metabolite markers may reflect certain manifestations; for example, Hcy is negatively associated with cognitive performance. Fifth, alteration of microbial diversity and composition has been observed in multiple studies; therefore, it may reflect the presence of disease and predict the risk and disease stage of schizophrenia. In addition, the gut microbiota has been shown to differentiate between acute schizophrenia and remission and between deficit and non-deficit schizophrenia. Finally, pharmacogenomic evaluations are critical for individualised treatment strategies, as higher polygenic risk scores predict greater post-treatment symptoms, whereas lower polygenic risk scores indicate a higher possibility of response to treatment. It is noteworthy that polymorphism of the dopamine transporter gene, rs2975226, has been identified in a Chinese schizophrenia cohort as an indicator of responsiveness to clozapine, which may be used as a population-specific marker.

\section{CONCLUSIONS}

This study comprehensively and systematically analysed potential biomarkers for identifying schizophrenia, providing a first-hand laboratory testing basis for clinical diagnosis. With the continuous development of new 
diagnostic techniques, we believe that more biomarkers will be added to this consensus in the near future.

\section{FUTURE DIRECTIONS}

Although genetic risk factors play an important role in developing schizophrenia, further investigations and validations in larger cohorts of certain ethnic groups are required before genetic markers can be used as a diagnostic tool for schizophrenia. This is because novel loci have been identified in a variety of studies, and these loci both overlap and vary across different ethnicities. Epigenetic markers such as DNA methylation sites and miRNAs may be useful biomarkers, but the inconsistency of results generated from different studies makes it difficult to develop specific diagnostic markers. Further investigations are needed to identify schizophrenia-specific genetic markers for precise diagnosis, differential diagnosis and ethnicity-specific markers in the Chinese population. Analysing appropriate biospecimens for accurate detection of genetic markers is also challenging, as peripheral blood mononuclear cells have been used in studies of schizophrenia-associated miRNAs. In future studies, we need to determine an optimised method and sample type to be used for developing diagnostic biomarkers. In terms of neurotransmitters, most of the studies are established by analysing in vivo imaging or postmortem brain tissue and occasionally CSF. Assessment of neurotransmitters and their metabolites in peripheral biofluids, particularly in the blood, is lacking. The development of certain novel technologies such as single-molecule array technology and nanobiotechnology is making the clinical use of markers such as disease diagnostic biomarkers possible. In addition, the consistency of the data generated from different studies on the gut microbiota is not satisfactory. In future research, apart from transverse analysis, longitudinal comparison using each individual's data and randomized controlled trials (RCT) are critical for the prediction of disease progression and effectiveness of interventions.

\section{Author affiliations}

${ }^{1}$ Department of Clinical Laboratory, Shanghai Mental Health Center, Shanghai Jiao Tong University School of Medicine, Shanghai, China

${ }^{2}$ Department of Medical Microbiology and Parasitology, Fudan University School of Basic Medical Sciences, Shanghai, China

${ }^{3}$ Department of Psychosis, Institute of Psychiatry, Psychology and Neuroscience, King's College London, London, UK

${ }^{4}$ Clinical Laboratory, Affiliated Hospital of West Anhui Health Vocational College, Lu'an, Anhui, China

${ }^{5}$ School of Global Health, Chinese Center for Tropical Diseases Research, Shanghai Jiao Tong University School of Medicine, Shanghai, China

${ }^{6}$ Editorial Office of General Psychiatry, Shanghai Mental Health Center, Shanghai Jiao Tong University School of Medicine, Shanghai, China

${ }^{7}$ Shanghai Key Laboratory of Psychotic Disorders, Shanghai Jiao Tong University School of Medicine, Shanghai, China

Acknowledgements We thank Dr Yang Sun from Institute of Arthritis Research, Shanghai Academy of Chinese Medical Sciences for critical review of this manuscript.
Contributors The paper was co-authored by PL, JS, XL, et al. These authors have contributed equally to this work. JC, $X C$ and $X G$ are joint corresponding authors in this work. Manuscript concept and design: XG, XC, JC, PL, XL, JS. Manuscript drafting: PL, XL, JS. Critical review of literatures: PL, XL, JS, DL, YS, ZL, PM, PLi, SC, WJ, SL, QC, QG, LZ, JX, MZ, MW, KL, LZ, HX, KD, QL. Critical revision of the manuscript: XG, XC, JC, PL. All co-authors contributed to this manuscript and agreed to the final submission.

Funding This study was funded by Shanghai Natural Science fund Project under grant (20ZR1447700), The National Key Development Plan for Precision Medicine Research (2017YFC0910002), the hospital-level project of Shanghai Mental Health Center under grant (2020-YJ05), Special Fund for Clinical Research of Wu Jieping Medical Foundation (320675015232), the Hospital level project of Shanghai Mental Health Center (2016-YJ-09), Chinese medicine research project of Shanghai Health and Family Planning Commission (2018LP024), the characteristic discipline construction project of Shanghai Mental Health Center (2017-TSXK-07), China Public Health Alliance (first batch) (GWLM202014).

Competing interests None declared.

Patient consent for publication Not applicable.

Ethics approval This study does not involve human participants.

Provenance and peer review Commissioned; externally peer reviewed.

Data availability statement All data relevant to the study are included in the article or uploaded as supplementary information.

Supplemental material This content has been supplied by the author(s). It has not been vetted by BMJ Publishing Group Limited (BMJ) and may not have been peer-reviewed. Any opinions or recommendations discussed are solely those of the author(s) and are not endorsed by BMJ. BMJ disclaims all liability and responsibility arising from any reliance placed on the content. Where the content includes any translated material, BMJ does not warrant the accuracy and reliability of the translations (including but not limited to local regulations, clinical guidelines, terminology, drug names and drug dosages), and is not responsible for any error and/or omissions arising from translation and adaptation or otherwise.

Open access This is an open access article distributed in accordance with the Creative Commons Attribution Non Commercial (CC BY-NC 4.0) license, which permits others to distribute, remix, adapt, build upon this work non-commercially, and license their derivative works on different terms, provided the original work is properly cited, appropriate credit is given, any changes made indicated, and the use is non-commercial. See: http://creativecommons.org/licenses/by-nc/4.0/.

ORCID iD

Jinghong Chen http://orcid.org/0000-0002-7428-9359

\section{REFERENCES}

1 Owen MJ, Sawa A, Mortensen PB. Schizophrenia. Lancet 2016;388:86-97.

2 Javitt DC. Balancing therapeutic safety and efficacy to improve clinical and economic outcomes in schizophrenia: a clinical overview. Am J Manag Care 2014;20:S160-5.

3 McGrath JJ. Variations in the incidence of schizophrenia: data versus dogma. Schizophr Bull 2006;32:195-7.

4 Torrey EF, Miller J, Rawlings R, et al. Seasonality of births in schizophrenia and bipolar disorder: a review of the literature. Schizophr Res 1997;28:1-38.

5 Brown AS, Hooton J, Schaefer CA, et al. Elevated maternal interleukin-8 levels and risk of schizophrenia in adult offspring. Am J Psychiatry 2004;161:889-95.

6 Thibaut F. Schizophrenia: an example of complex genetic disease. World J Biol Psychiatry 2006;7:194-7.

7 Soares-Weiser K, Maayan N, Bergman H, et al. First rank symptoms for schizophrenia. Cochrane Database Syst Rev 2015;1:CD010653.

8 Foussias G, Remington G. Negative symptoms in schizophrenia: avolition and Occam's razor. Schizophr Bull 2010;36:359-69.

9 van de Leemput J, Hess JL, Glatt SJ, et al. Genetics of schizophrenia: historical insights and prevailing evidence. $A d v$ Genet 2016;96:99-141.

10 Picchioni MM, Murray RM. Schizophrenia. BMJ 2007;335:91-5.

11 Nakahara S, Medland S, Turner JA, et al. Polygenic risk score, genome-wide association, and gene set analyses of cognitive domain deficits in schizophrenia. Schizophr Res 2018;201:393-9.

12 Addington AM, Rapoport JL. The genetics of childhood-onset schizophrenia: when madness strikes the prepubescent. Curr Psychiatry Rep 2009;11:156-61. 
13 International Schizophrenia Consortium, Purcell SM, Wray NR, et al. Common polygenic variation contributes to risk of schizophrenia and bipolar disorder. Nature 2009;460:748-52.

14 McCutcheon RA, Reis Marques T, Howes OD. Schizophrenia-an overview. JAMA Psychiatry 2020;77:201-10.

15 Murphy KC. Schizophrenia and velo-cardio-facial syndrome. Lancet 2002;359:426-30.

16 Sporn A, Addington A, Reiss AL, et al. 22Q11 deletion syndrome in childhood onset schizophrenia: an update. Mol Psychiatry 2004;9:225-6.

17 Marshall CR, Howrigan DP, Merico D, et al. Contribution of copy number variants to schizophrenia from a genome-wide study of 41,321 subjects. Nat Genet 2017;49:27-35.

18 Murphy KC, Jones LA, Owen MJ. High rates of schizophrenia in adults with velo-cardio-facial syndrome. Arch Gen Psychiatry 1999;56:940-5

19 Bassett AS, Chow EWC. Schizophrenia and 22q11.2 deletion syndrome. Curr Psychiatry Rep 2008;10:148-57.

20 Lowther C, Costain G, Baribeau DA, et al. Genomic disorders in Psychiatry-what does the clinician need to know? Curr Psychiatry Rep 2017;19:82.

21 Lam M, Chen C-Y, Li Z, et al. Comparative genetic architectures of schizophrenia in East Asian and European populations. Nat Genet 2019;51:1670-8.

22 Schizophrenia Working Group of the Psychiatric Genomics Consortium. Biological insights from 108 schizophrenia-associated genetic loci. Nature 2014;511:421-7.

23 Psychiatric GWAS Consortium Coordinating Committee, Cichon S, Craddock N, et al. Genomewide association studies: history, rationale, and prospects for psychiatric disorders. Am J Psychiatry 2009:166:540-56.

24 Schizophrenia Psychiatric Genome-Wide Association Study (GWAS) Consortium. Genome-wide association study identifies five new schizophrenia loci. Nat Genet 2011;43:969-76

25 Rietschel M, Mattheisen M, Degenhardt F, et al. Association between genetic variation in a region on chromosome 11 and schizophrenia in large samples from Europe. Mol Psychiatry 2012;17:906-17.

26 Cross-Disorder Group of the Psychiatric Genomics Consortium Identification of risk loci with shared effects on five major psychiatric disorders: a genome-wide analysis. Lancet 2013;381:1371-9.

27 Irish Schizophrenia Genomics Consortium and the Wellcome Trust Case Control Consortium 2. Genome-wide association study implicates HLA-C ${ }^{\star}$ 01:02 as a risk factor at the major histocompatibility complex locus in schizophrenia. Biol Psychiatry 2012;72:620-8.

28 Hamshere ML, Walters JTR, Smith R, et al. Genome-wide significant associations in schizophrenia to ITIH3/4, CACNA1C and SDCCAG8, and extensive replication of associations reported by the schizophrenia PGC. Mol Psychiatry 2013;18:708-12.

29 Ripke S, O'Dushlaine C, Chambert K, et al. Genome-wide association analysis identifies 13 new risk loci for schizophrenia. Nat Genet 2013;45:1150-9.

30 Stefansson H, Ophoff RA, Steinberg S, et al. Common variants conferring risk of schizophrenia. Nature 2009;460:744-7.

31 O'Donovan MC, Craddock N, Norton N, et al. Identification of loci associated with schizophrenia by genome-wide association and follow-up. Nat Genet 2008;40:1053-5.

32 Hartwig FP, Bowden J, Loret de Mola C, et al. Body mass index and psychiatric disorders: a Mendelian randomization study. Sci Rep 2016;6:32730

$33 \mathrm{Li} \mathrm{Z,} \mathrm{Chen} \mathrm{J,} \mathrm{Yu} \mathrm{H,} \mathrm{et} \mathrm{al.} \mathrm{Genome-wide} \mathrm{association} \mathrm{analysis}$ identifies 30 new susceptibility loci for schizophrenia. Nat Genet 2017;49:1576-83.

34 Ikeda M, Aleksic B, Kinoshita Y, et al. Genome-wide association study of schizophrenia in a Japanese population. Biol Psychiatry 2011;69:472-8.

35 Cassidy C, Buchy L, Bodnar M, et al. Association of a risk allele of ANK3 with cognitive performance and cortical thickness in patients with first-episode psychosis. J Psychiatry Neurosci 2014;39:31-9.

36 Gurung R, Prata DP. What is the impact of genome-wide supported risk variants for schizophrenia and bipolar disorder on brain structure and function? A systematic review. Psychol Med 2015;45:2461-80.

37 Cho Y, Ryu S, Huh I, et al. Effects of genetic variations in NRG1 on cognitive domains in patients with schizophrenia and healthy individuals. Psychiatr Genet 2015;25:147-54.

38 Matosin N, Newell KA, Quidé Y, et al. Effects of common GRM5 genetic variants on cognition, hippocampal volume and
mGluR5 protein levels in schizophrenia. Brain Imaging Behav 2018;12:509-17.

39 Baek JH, Kim JS, Ryu S, et al. Association of genetic variations in DTNBP1 with cognitive function in schizophrenia patients and healthy subjects. Am J Med Genet B Neuropsychiatr Genet 2012;159B:841-9.

40 Varela-Gomez N, Mata I, Perez-Iglesias R, et al. Dysbindin gene variability is associated with cognitive abnormalities in first-episode non-affective psychosis. Cogn Neuropsychiatry 2015;20:144-56.

41 Vyas NS, Lee Y, Ahn K, et al. Association of a serotonin receptor 2A gene polymorphism with visual sustained attention in early-onset schizophrenia patients and their non-psychotic siblings. Aging Dis 2012;3:291-300.

42 Sun Z, Ma Y, Li W, et al. Associations between the DBH gene, plasma dopamine $\beta$-hydroxylase activity and cognitive measures in Han Chinese patients with schizophrenia. Schizophr Res 2018;193:58-63.

43 Yates D. Synaptic plasticity: micro-level disruption. Nat Rev Neurosci 2015;16:373.

$44 \mathrm{Kim}$ AH, Parker EK, Williamson V, et al. Experimental validation of candidate schizophrenia gene ZNF804A as target for hsa-miR-137. Schizophr Res 2012;141:60-4

45 Kwon E, Wang W, Tsai L-H. Validation of schizophrenia-associated genes CSMD1, C10orf26, CACNA1C and TCF4 as miR-137 targets. Mol Psychiatry 2013;18:11-12.

46 Corvin A, Morris DW. Genome-wide association studies: findings at the major histocompatibility complex locus in psychosis. Biol Psychiatry 2014;75:276-83.

47 Egan MF, Goldberg TE, Kolachana BS, et al. Effect of COMT Val108/158 Met genotype on frontal lobe function and risk for schizophrenia. Proc Natl Acad Sci U S A 2001;98:6917-22.

48 Skene NG, Bryois J, Bakken TE, et al. Genetic identification of brain cell types underlying schizophrenia. Nat Genet 2018;50:825-33.

49 Cross-Disorder Group of the Psychiatric Genomics Consortium, Lee SH, Ripke S, et al. Genetic relationship between five psychiatric disorders estimated from genome-wide SNPs. Nat Genet 2013;45:984-94.

50 Pries L-K, Gülöksüz S, Kenis G. DNA methylation in schizophrenia. Adv Exp Med Biol 2017;978:211-36.

51 Abdolmaleky HM, Cheng K-hung, Russo A, et al. Hypermethylation of the Reelin (RELN) promoter in the brain of schizophrenic patients: a preliminary report. Am J Med Genet B Neuropsychiatr Genet 2005;134B:60-6.

52 Huang H-S, Akbarian S. Gad1 mRNA expression and DNA methylation in prefrontal cortex of subjects with schizophrenia. PLoS One 2007;2:e809.

53 Carrard A, Salzmann A, Malafosse A, et al. Increased DNA methylation status of the serotonin receptor 5 HTR1A gene promoter in schizophrenia and bipolar disorder. J Affect Disord 2011;132:450-3.

54 Li M, Li Y, Qin H, et al. Genome-Wide DNA methylation analysis of peripheral blood cells derived from patients with first-episode schizophrenia in the Chinese Han population. Mol Psychiatry 2021;26:4475-4485.

55 Perkins DO, Jeffries CD, Jarskog LF, et al. microRNA expression in the prefrontal cortex of individuals with schizophrenia and schizoaffective disorder Genome Biol 2007:8:R27.

56 Santarelli DM, Beveridge NJ, Tooney PA, et al. Upregulation of Dicer and microRNA expression in the dorsolateral prefrontal cortex Brodmann area 46 in schizophrenia. Biol Psychiatry 2011;69:180-7.

57 Liu S, Zhang F, Wang X, et al. Diagnostic value of blood-derived microRNAs for schizophrenia: results of a meta-analysis and validation. Sci Rep 2017;7:15328

58 Izumi R, Hino M, Wada A, et al. Detailed postmortem profiling of inflammatory mediators expression revealed post-inflammatory alternation in the superior temporal gyrus of schizophrenia. Front Psychiatry 2021;12:653821.

59 Orsolini L, Sarchione F, Vellante F, et al. Protein- $C$ reactive as biomarker predictor of schizophrenia phases of illness? A systematic review. Curr Neuropharmacol 2018;16:583-606.

60 Sanada K, Montero-Marin J, Barceló-Soler A, et al. Effects of mindfulness-based interventions on biomarkers and low-grade inflammation in patients with psychiatric disorders: a meta-analytic review. Int J Mol Sci 2020;21. doi:10.3390/ijms21072484. [Epub ahead of print: 03 Apr 2020].

61 Bora E. Peripheral inflammatory and neurotrophic biomarkers of cognitive impairment in schizophrenia: a meta-analysis. Psychol Med 2019;49:1971-9.

62 Frydecka D, Krzystek-Korpacka M, Lubeiro A, et al. Profiling inflammatory signatures of schizophrenia: a cross-sectional and meta-analysis study. Brain Behav Immun 2018;71:28-36. 
63 Reale M, Costantini E, Greig NH. Cytokine imbalance in schizophrenia. from research to clinic: potential implications for treatment. Front Psychiatry 2021;12:536257.

64 Orlovska-Waast S, Köhler-Forsberg O, Brix SW, et al. Cerebrospinal fluid markers of inflammation and infections in schizophrenia and affective disorders: a systematic review and meta-analysis. Mol Psychiatry 2019;24:869-87.

65 Feng T, McEvoy JP, Miller BJ. Longitudinal study of inflammatory markers and psychopathology in schizophrenia. Schizophr Res 2020;224:58-66.

66 Joaquim HPG, Costa AC, Gattaz WF, et al. Kynurenine is correlated with IL $-1 \beta$ in plasma of schizophrenia patients. J Neural Transm 2018;125:869-73.

67 Pedraz-Petrozzi B, Elyamany O, Rummel C, et al. Effects of inflammation on the kynurenine pathway in schizophrenia - a systematic review. J Neuroinflammation 2020;17:56.

68 Miller BJ, Buckley P, Seabolt W, et al. Meta-analysis of cytokine alterations in schizophrenia: clinical status and antipsychotic effects. Biol Psychiatry 2011;70:663-71.

69 Eaton WW, Byrne M, Ewald H, et al. Association of schizophrenia and autoimmune diseases: linkage of Danish national registers. Am J Psychiatry 2006;163:521-8.

70 Wright P, Sham PC, Gilvarry CM, et al. Autoimmune diseases in the pedigrees of schizophrenic and control subjects. Schizophr Res 1996;20:261-7.

71 Benros ME, Nielsen PR, Nordentoft M, et al. Autoimmune diseases and severe infections as risk factors for schizophrenia: a 30-year population-based register study. Am J Psychiatry 2011;168:1303-10

72 Chen S-J, Chao Y-L, Chen C-Y, et al. Prevalence of autoimmune diseases in in-patients with schizophrenia: nationwide populationbased study. Br J Psychiatry 2012;200:374-80.

73 Tiosano S, Farhi A, Watad A, et al. Schizophrenia among patients with systemic lupus erythematosus: population-based crosssectional study. Epidemiol Psychiatr Sci 2017;26:424-9.

74 Sellgren C, Frisell T, Lichtenstein P, et al. The association between schizophrenia and rheumatoid arthritis: a nationwide populationbased Swedish study on intraindividual and familial risks. Schizophr Bull 2014;40:1552-9.

75 Stahl SM. Beyond the dopamine hypothesis of schizophrenia to three neural networks of psychosis: dopamine, serotonin, and glutamate. CNS Spectr 2018;23:187-91.

76 Meltzer HY, Stahl SM. The dopamine hypothesis of schizophrenia: a review. Schizophr Bull 1976;2:19-76.

77 Rasmussen H, Erritzoe D, Andersen R, et al. Decreased frontal serotonin2A receptor binding in antipsychotic-naive patients with first-episode schizophrenia. Arch Gen Psychiatry 2010;67:9-16.

78 Rasmussen H, Frokjaer VG, Hilker RW, et al. Low frontal serotonin $2 \mathrm{~A}$ receptor binding is a state marker for schizophrenia? Eur Neuropsychopharmacol 2016;26:1248-50.

79 Bleich A, Brown SL, Kahn R, et al. The role of serotonin in schizophrenia. Schizophr Bull 1988;14:297-315.

80 Lindström LH. Low HVA and normal 5HIAA CSF levels in drugfree schizophrenic patients compared to healthy volunteers: correlations to symptomatology and family history. Psychiatry Res 1985;14:265-73.

81 Carlborg A, Jokinen J, Nordström A-L, et al. Csf 5-HIAA, attempted suicide and suicide risk in schizophrenia spectrum psychosis. Schizophr Res 2009;112:80-5.

82 Abi-Dargham A, Gil R, Krystal J, et al. Increased striatal dopamine transmission in schizophrenia: confirmation in a second cohort. Am J Psychiatry 1998;155:761-7.

83 Ruhrmann S, Bechdolf A, Kühn K-U, et al. Acute effects of treatment for prodromal symptoms for people putatively in a late initial prodromal state of psychosis. Br J Psychiatry Supp 2007;51:s88-95.

84 Seeman P, Kapur S. Schizophrenia: more dopamine, more D2 receptors. Proc Natl Acad Sci U S A 2000;97:7673-5.

85 Slifstein M, van de Giessen E, Van Snellenberg J, et al. Deficits in prefrontal cortical and extrastriatal dopamine release in schizophrenia: a positron emission tomographic functional magnetic resonance imaging study. JAMA Psychiatry 2015;72:316-24.

86 Wieselgren IM, Lindström LH. Csf levels of HVA and 5-HIAA in drug-free schizophrenic patients and healthy controls: a prospective study focused on their predictive value for outcome in schizophrenia. Psychiatry Res 1998;81:101-10.

87 Howes OD, Williams M, Ibrahim K, et al. Midbrain dopamine function in schizophrenia and depression: a post-mortem and positron emission tomographic imaging study. Brain 2013:136:3242-51.
88 Howes O, McCutcheon R, Stone J. Glutamate and dopamine in schizophrenia: an update for the 21st century. J Psychopharmacol 2015;29:97-115.

89 Howes OD, Kambeitz J, Kim E, et al. The nature of dopamine dysfunction in schizophrenia and what this means for treatment. Arch Gen Psychiatry 2012;69:776-86.

90 Howes OD, Montgomery AJ, Asselin M-C, et al. Elevated striatal dopamine function linked to prodromal signs of schizophrenia. Arch Gen Psychiatry 2009;66:13-20.

91 Merritt K, Egerton A, Kempton MJ, et al. Nature of glutamate alterations in schizophrenia: a meta-analysis of proton magnetic resonance spectroscopy studies. JAMA Psychiatry 2016;73:665-74.

92 Kumar J, Liddle EB, Fernandes CC, et al. Glutathione and glutamate in schizophrenia: a 7T MRS study. Mol Psychiatry 2020;25:873-82.

93 Coughlin JM, Yang K, Marsman A, et al. A multimodal approach to studying the relationship between peripheral glutathione, brain glutamate, and cognition in health and in schizophrenia. Mol Psychiatry 2021;26:3502-3511.

94 Catak Z, Kocdemir E, Ugur K, et al. A novel biomarker renalase and its relationship with its substrates in schizophrenia. J Med Biochem 2019;38:299-305

95 Savransky A, Chiappelli J, Du X, et al. Association of working memory and elevated overnight urinary norepinephrine in patients with schizophrenia. J Psychiatr Res 2021;137:89-95.

96 Qin X-Y, Wu H-T, Cao C, et al. A meta-analysis of peripheral blood nerve growth factor levels in patients with schizophrenia. $\mathrm{Mol}$ Psychiatry 2017;22:1306-12.

97 Rao S, Martínez-Cengotitabengoa M, Yao Y, et al. Peripheral blood nerve growth factor levels in major psychiatric disorders. J Psychiatr Res 2017;86:39-45.

98 Chu C-S, Chu C-L, Wu C-C, et al. Serum nerve growth factor beta, brain- and glial-derived neurotrophic factor levels and psychopathology in unmedicated patients with schizophrenia. $J$ Chin Med Assoc 2018;81:577-81.

99 Çakici N, Sutterland AL, Penninx BWJH, et al. Altered peripheral blood compounds in drug-naïve first-episode patients with either schizophrenia or major depressive disorder: a meta-analysis. Brain Behav Immun 2020;88:547-58.

100 Neugebauer K, Hammans C, Wensing T, et al. Nerve growth factor serum levels are associated with regional gray matter volume differences in schizophrenia patients. Front Psychiatry 2019;10:275

101 Yang Y, Liu Y, Wang G, et al. Brain-Derived neurotrophic factor is associated with cognitive impairments in first-episode and chronic schizophrenia. Psychiatry Res 2019;273:528-36.

102 Fang X, Chen Y, Wang Y, et al. Depressive symptoms in schizophrenia patients: a possible relationship between SIRT1 and BDNF. Prog Neuropsychopharmacol Biol Psychiatry 2019;95:109673.

103 Heitz U, Papmeyer M, Studerus E, et al. Plasma and serum brainderived neurotrophic factor (BDNF) levels and their association with neurocognition in at-risk mental state, first episode psychosis and chronic schizophrenia patients. World J Biol Psychiatry 2019;20:545-54.

104 Weickert CS, Lee CH, Lenroot RK, et al. Increased plasma brain-derived neurotrophic factor (BDNF) levels in females with schizophrenia. Schizophr Res 2019;209:212-7.

105 Agrawal A, llango K, Singh PK, et al. Age dependent levels of plasma homocysteine and cognitive performance. Behav Brain Res 2015;283:139-44

106 Yang Y, Wang J, Xiong Z, et al. Prevalence and clinical demography of hyperhomocysteinemia in Han Chinese patients with schizophrenia. Eur Arch Psychiatry Clin Neurosci 2021;271:759-65.

107 Trześniowska-Drukała B, Kalinowska S, Safranow K, et al. Evaluation of hyperhomocysteinemia prevalence and its influence on the selected cognitive functions in patients with schizophrenia. Prog Neuropsychopharmacol Biol Psychiatry 2019;95:109679.

108 Liu Y, Tao H, Yang X, et al. Decreased serum oxytocin and increased homocysteine in first-episode schizophrenia patients. Front Psychiatry 2019;10:217.

109 Zhang Y, Zhao J, Wang W, et al. Homocysteine, but not MTHFR gene polymorphism, influences depressive symptoms in patients with schizophrenia. J Affect Disord 2020;272:24-7.

110 Kinoshita M, Numata S, Tajima A, et al. Cumulative effect of the plasma total homocysteine-related genetic variants on schizophrenia risk. Psychiatry Res 2016;246:833-7.

111 Fryar-Williams S, Strobel JE. Biomarkers of a five-domain translational substrate for schizophrenia and schizoaffective psychosis. Biomark Res 2015;3:3: 3.

112 Cao B, Sun X-Y, Zhang C-B, et al. Association between B vitamins and schizophrenia: a population-based case-control study. Psychiatry Res 2018;259:501-5. 
113 Tomioka Y, Numata S, Kinoshita M, et al. Decreased serum pyridoxal levels in schizophrenia: meta-analysis and Mendelian randomization analysis. J Psychiatry Neurosci 2018;43:194-200.

114 Yazici AB, Akcay Ciner O, Yazici E, et al. Comparison of vitamin $B 12$, vitamin $D$ and folic acid blood levels in patients with schizophrenia, drug addiction and controls. J Clin Neurosci 2019;65:11-16.

115 Ishiwata S, Hattori K, Sasayama D, et al. Plasma and cerebrospinal fluid G72 protein levels in schizophrenia and major depressive disorder. Psychiatry Res 2017;254:244-50.

116 Lin E, Lin C-H, Lai Y-L, et al. Combination of G72 genetic variation and G72 protein level to detect schizophrenia: machine learning approaches. Front Psychiatry 2018;9:566.

117 Akyol ES, Albayrak Y, Aksoy N, et al. Increased serum G72 protein levels in patients with schizophrenia: a potential candidate biomarker. Acta Neuropsychiatr 2017;29:80-6.

118 Jagannath V, Gerstenberg M, Correll CU, et al. A systematic metaanalysis of the association of neuregulin 1 (NRG1), D-amino acid oxidase (DAO), and DAO activator (DAOA)/G72 polymorphisms with schizophrenia. J Neural Transm 2018;125:89-102.

119 Nguyen TT, Kosciolek T, Eyler LT, et al. Overview and systematic review of studies of microbiome in schizophrenia and bipolar disorder. J Psychiatr Res 2018:99:50-61.

120 Zhu F, Ju Y, Wang W, et al. Metagenome-wide association of gut microbiome features for schizophrenia. Nat Commun 2020;11:1612.

121 Shen Y, Xu J, Li Z, et al. Analysis of gut microbiota diversity and auxiliary diagnosis as a biomarker in patients with schizophrenia: a cross-sectional study. Schizophr Res 2018;197:470-7.

122 Wu J, Li J, Gaurav C, et al. CUMS and dexamethasone induce depression-like phenotypes in mice by differentially altering gut microbiota and triggering macroglia activation. Gen Psychiatr 2021;34:e100529.

$123 \mathrm{Ma} \mathrm{X}$, Asif H, Dai L, et al. Alteration of the gut microbiome in firstepisode drug-naïve and chronic medicated schizophrenia correlate with regional brain volumes. J Psychiatr Res 2020;123:136-44.

$124 \mathrm{Xu}$ R, Wu B, Liang J, et al. Altered gut microbiota and mucosal immunity in patients with schizophrenia. Brain Behav Immun 2020;85:120-7.

125 Yuan X, Zhang P, Wang Y, et al. Changes in metabolism and microbiota after 24-week risperidone treatment in drug naïve, normal weight patients with first episode schizophrenia. Schizophr Res 2018;201:299-306.

126 Zhuang Z, Yang R, Wang W, et al. Associations between gut microbiota and Alzheimer's disease, major depressive disorder, and schizophrenia. J Neuroinflammation 2020;17:288.

127 Nguyen TT, Kosciolek T, Daly RE, et al. Gut microbiome in schizophrenia: altered functional pathways related to immune modulation and atherosclerotic risk. Brain Behav Immun 2021;91:245-56

128 Zhu C, Zheng M, Ali U. Association between abundance of Haemophilus in the gut microbiota and negative symptoms of schizophrenia. Front Psychiatry 2021;12:685910.

129 Maes M, Kanchanatawan B, Sirivichayakul S, et al. In schizophrenia, increased plasma IgM/lgA responses to gut commensal bacteria are associated with negative symptoms, neurocognitive impairments, and the deficit phenotype. Neurotox Res 2019;35:684-98.

130 Severance EG, Gressitt KL, Stallings CR, et al. Probiotic normalization of Candida albicans in schizophrenia: a randomized, placebo-controlled, longitudinal pilot study. Brain Behav Immun 2017;62:41-5

131 Bousman CA, Bengesser SA, Aitchison KJ, et al. Review and consensus on pharmacogenomic testing in psychiatry. Pharmacopsychiatry 2021;54:5-17.

132 Zhang J-P, Robinson D, Yu J, et al. Schizophrenia polygenic risk score as a predictor of antipsychotic efficacy in first-episode psychosis. Am J Psychiatry 2019;176:21-8.

133 Xu M, Xing Q, Li S, et al. Pharacogenetic effects of dopamine transporter gene polymorphisms on response to chlorpromazine and clozapine and on extrapyramidal syndrome in schizophrenia. Prog Neuropsychopharmacol Biol Psychiatry 2010;34:1026-32.

134 Arranz MJ, Munro J, Birkett J, et al. Pharmacogenetic prediction of clozapine response. Lancet 2000;355:1615-6.

135 Yang B, Wei J, Ju P, et al. Effects of regulating intestinal microbiota on anxiety symptoms: a systematic review. Gen Psychiatr 2019;32:e100056.

$136 \mathrm{Li} \mathrm{J}$, Hashimoto H, Meltzer HY. Association of serotonin2 $\mathrm{c}_{2 \mathrm{c}}$ receptor polymorphisms with antipsychotic drug response in schizophrenia. Front Psychiatry 2019;10:58.

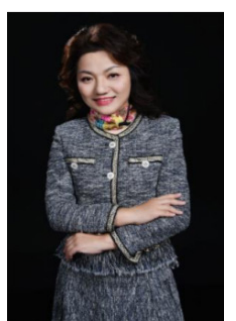

Ping Lin obtained a master's degree from Shanghai Jiao Tong University School of Medicine, China, in 2010. Now she has been working on a PhD program at Fudan University School of Medicine since 2017. She is currently working as the director of technician at the department of clinical laboratory in Shanghai Mental Health Center in China. She is also the director of the expert committee of Sprit and Mental Illness on the Chinese Medical Doctor Association, Branch of Clinical Laboratory Physician, the leader of the accurate inspection group of the mental illness on Chinese research institute, hospital inspection medical professional committee, and the editor of different journals: Laboratory Medicine, The International Journal of Laboratory Medicine, and Frontiers in Laboratory Medicine. Her main research interest includes the new method of clinical laboratory in diagnosing mental illness. 